\title{
Acute Disseminated Encephalopathy Combined With Anticentromere Antibody B Positive: Uncommon Primary Manifestations of Sjogren's Syndrome
}

\author{
Jianfeng $\mathrm{Qu}^{\mathrm{a}, \mathrm{b}}$, Yankun Chen ${ }^{\mathrm{a}}$
}

\begin{abstract}
Sjogren's syndrome (SS) can involve the central nervous system; however, acute disseminated encephalopathy has rarely been reported as the initial manifestation. We report a 60 -year-old woman with SS and anticentromere antibody B positive, presenting with acute disseminated encephalopathy. The diagnosis of SS was based on the presence of ocular staining score and labial salivary gland biopsies. SS can present with variable central nervous system signs and anticentromere antibody B positive, which may precede the classic sicca symptoms. Therefore, earlier recognition and treatment for this situation is important.
\end{abstract}

Keywords: Acute disseminated encephalopathy; Anticentromere antibodies B; Sjogren's syndrome

\section{Introduction}

Sjogren's syndrome (SS) is a common autoimmune disease, and has a reported incidence of $3.9-5.3 / 100,000$ inhabitants [1]. The disease is characterized by a chronic lymphocytic and plasma cellular infiltration of exocrine glands (autoimmune exocrinopathy) and extraglandular features including both peripheral nervous system (PNS) and central nervous system (CNS) involvement [2]. The main immunologic features of SS were positive serum anti-SSA and/or anti-SSB or positive rheumatoid factor and antinuclear antibody (ANA) $\geq 1: 320$ [3]. At the same time, the patients of SS can present anticentromere antibody B (ACA-B) positive [4]. CNS lesions in SS vary from diffuse involvement, which manifests as cognitive deficits or meningoencephalitis, to focal involvement, which presents with similar symptoms to multiple sclerosis, neuro-

\section{Manuscript accepted for publication June 24, 2016}

aDepartment of Neurology, Dongguan People's Hospital, Dongguan City, Guangdong Province, China

${ }^{b}$ Corresponding Author: Jianfeng Qu, Department of Neurology, Dongguan People's Hospital, Dongguan City, Guangdong Province, China.

Email: geoffrey-197830@163.com myelitis optica. Acute encephalopathy as the first presence is rare. We reported a patient of SS with ACA-B positive, presenting with acute disseminated encephalopathy.

\section{Case Report}

Because of progressive weakness and numbness of right limbs, a 60-year-old woman was admitted to the Department of Neurology, Dongguan People's Hospital, in September 2012. The patient had no history of hypertension, arthralgia, and xerostomia or xerophthalmia or cancers. On admission, her general examination was normal except forehead pachylosis (Fig. 1). The neurological examination revealed mild somnolence, right paralysis, bilateral Babinski's sign. The urgent brain CT (Fig. 2 ) showed multiple hypodensity lesions in the bilateral hemispheres.

The results of routine laboratory study including routine blood test, kidney function, myocardial enzyme, coagulation, liver function, thyroid, lipids, glucose, glycosylated hemoglobin, and C-reactive protein were at normal levels. Serological tests for hepatitis $\mathrm{C}$ virus, human immunodeficiency virus, syphilis and tumor markers were negative except HBsAg. The ANA was 1:100, ACA-B rather than anti-SSA and antiSSB bodies was positive, while other auto-antibodies were negative. The analysis of antineutrophil cytoplasmic antibody (ANCA) was normal. The brain magnetic resonance imaging (MRI) including diffusion-weighted image (DWI), perfusionweight image (PWI) and enhancement was performed the next

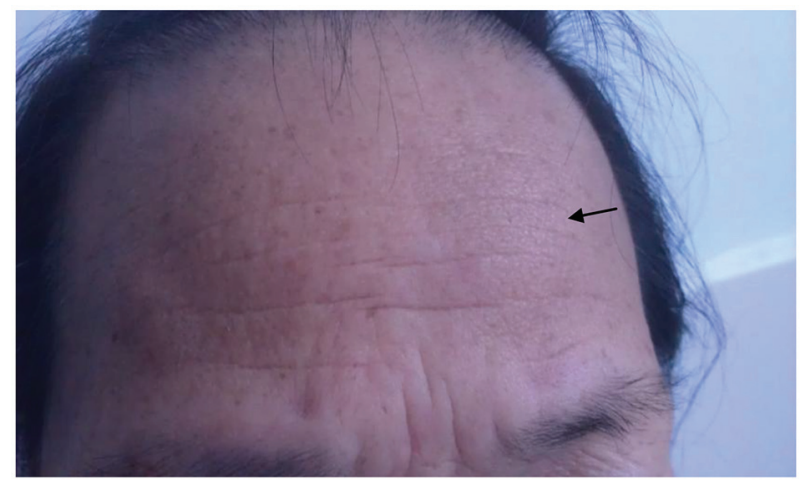

Figure 1. Forehead pachylosis (arrow). 


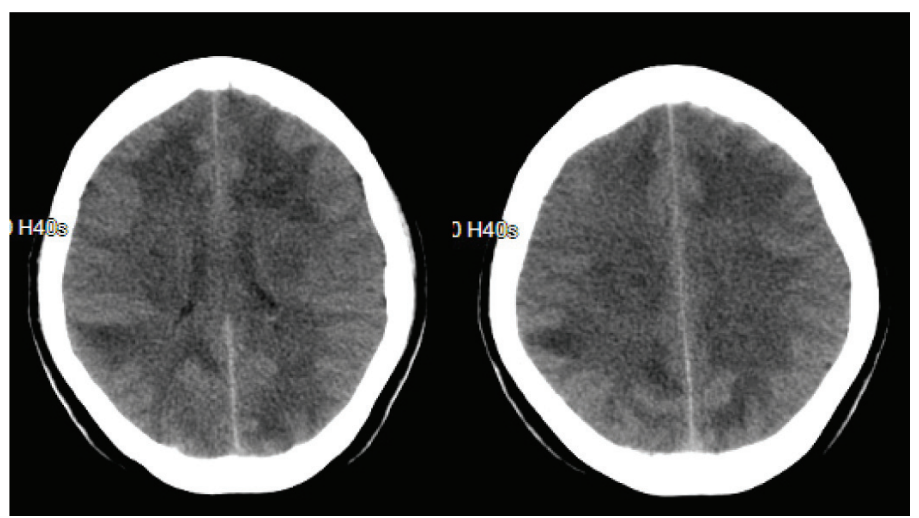

Figure 2. Brain CT obtained on the day of admission. The images show multiple hypodensity lesions in the bilateral hemispheres.

day after admission (Fig. 3). It showed supratentorial multiple lesions, surrounded by schistose brain edema, associated with some looping and nodal enhancement. DWI revealed some areas of restricted diffusion. PWI and magnetic resonance angiography (MRA) were feckly normal. Promptly, the level of consciousness had deteriorated. Lumbar puncture was performed 2 days after the admission, the pressure was $>330 \mathrm{~mm}$ $\mathrm{H}_{2} \mathrm{O}$, and the content was normal. Based on these data, the impression of acute disseminated encephalopathy was prompted. So methylprednisolone $1,000 \mathrm{mg} /$ day was administered by intravenous injection for 1 week. Her situation was improved in leaps and bounds. Because of the favorable recovery, the patient was discharged 20 days later. The methylprednisolone gradually tapered in the next 3 months.

And then she suffered xerostomia and xerophthalmia but no arthralgia gradually in the next 6 months. Unfortunately, she had no regard for it. These symptoms were progressive. She was admitted again 14 months later. Repeat brain MRI revealed that most of the lesions and hydrocephalus were remitted (Fig. 4). Analyses of auto-antibodies, ANCA, routine blood test, kidney function, myocardial enzyme, coagulation, liver function, thyroid, lipids, glucose, glycosylated hemoglobin, Creactive protein, serological tests for hepatitis $\mathrm{C}$ virus, human immunodeficiency virus, syphilis, HBsAg and tumor markers
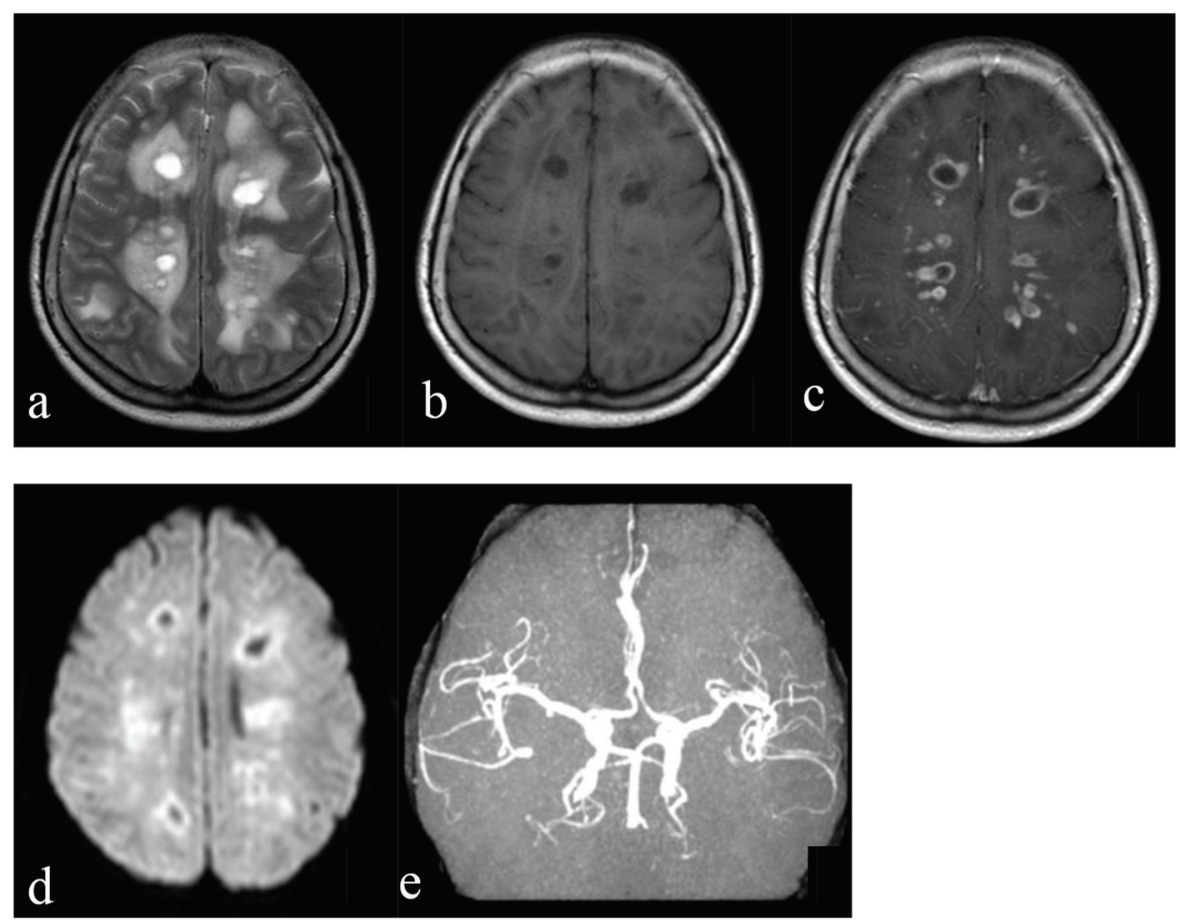

Figure 3. The first brain MRI after admission. (a) The sequence of T2-weighted image showed multiple hyperdensity round lesions in the bilateral hemispheres, mainly involved the white matter, which were surrounded by schistose brain edema. (b) The sequence of T1-weighted image revealed that the lesions were hypodensity. (c) The enhanced sequence showed looping and nodal enhancement. (d) DWI prompted some lesions were hyperdensity, which revealed restriction of diffusion. (e) MRA showed feckly normal. 


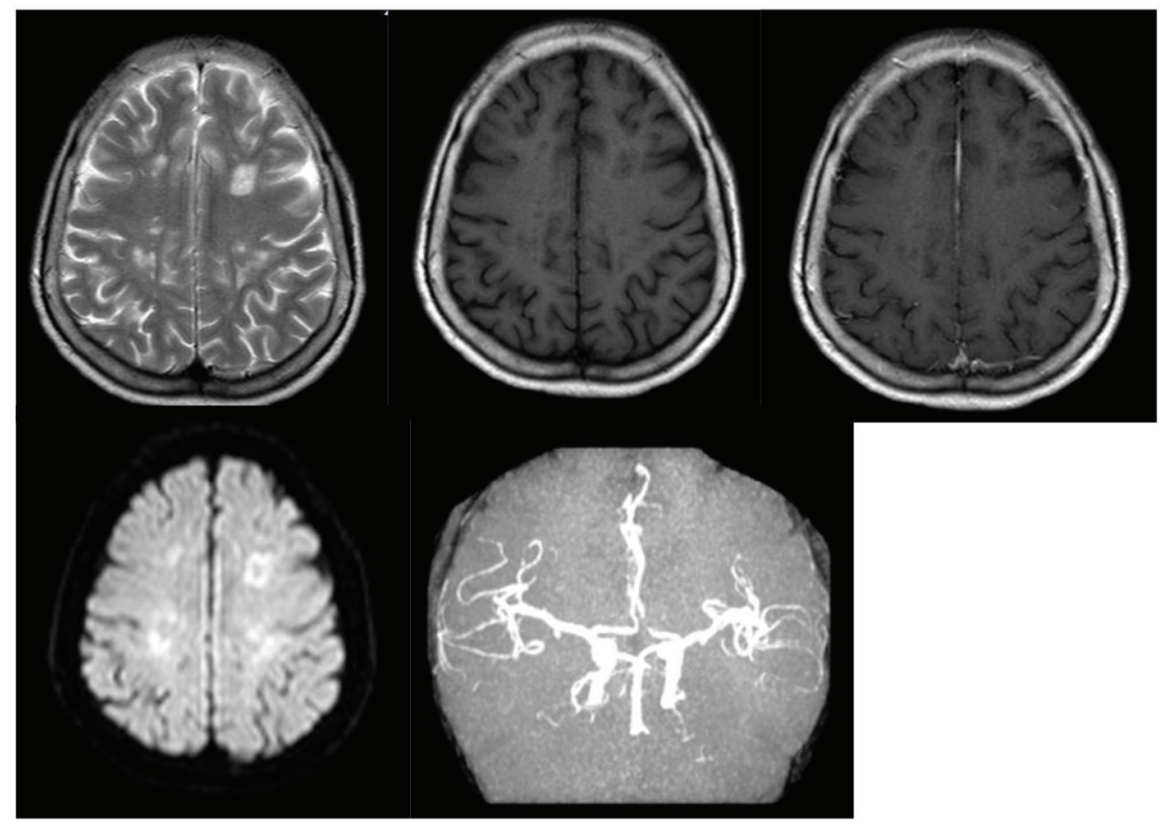

Figure 4. Repeated brain MRI revealed that most of the lesions and hydrocephalus were remitted.

were similar as the first time. In labial salivary gland biopsy, two foci of focal lymphocytic sialadenitis were present (Fig. 5); Schirmer's tear test results in both eyes were $<6 \mathrm{~mm}$ in 5 min (normal $>15 \mathrm{~mm})$; tear breakup time was $4 \mathrm{~s}$ (normal $\geq 10$ s); and punctate fluorescein staining was $>10$ (normal $<10)$. Based on the laboratory and biopsy findings, the patient was diagnosed with SS. Because her HBsAg was positive, we only prescribed hydroxychloroquine twice a day. And she did not relapse in the following 1.5 years.

\section{Discussion}

Clinical manifestations of neurological involvement in primary SS include PNS and CNS [5]. In an Italian cohort, the prevalence of CNS involvement was $5.8 \%$. Diffuse, focal $/$ multifocal, multiple sclerosis (MS)-like disease and isolated optic neuritis were the most common CNS clinical pictures [6]. Acute disseminated encephalopathy is rare. The patient we reported had severe diffuse white matter lesion, but good response to the methylprednisolone. She presented acute diffuse encephalopathy first, and the patient's ACA-B rather than anti-SSA and anti-SSB bodies was positive. Combined with the other symptoms, we considered about the systemic sclerosis (SSc). Then she suffered xerostomia and xerophthalmia subsequently. The ocular staining test and labial salivary gland biopsy were performed, though repeated MRI scan showed the lesions were remitted. According to the American College of Rheumatology Classification Criteria for Sjogren's Syndrome 2012 [3], the patient can be diagnosed as SS. In a research, prevalence of ACA among SS patients was 3.7\%, while the other was $4.7 \%[5,6]$. Clinical and immunological features of SS were largely similar among SS patients with ACA with and without SSc. However, the presence of ACA among patients with SS allows identification of a subset of patients with "SS overlap syndrome", who show a wide diversity of autoimmunity, encompassing but not limited to SSc $[6,7]$. Though the SS patients with ACA positive maybe show greater frequency of peripheral neuropathy, acute

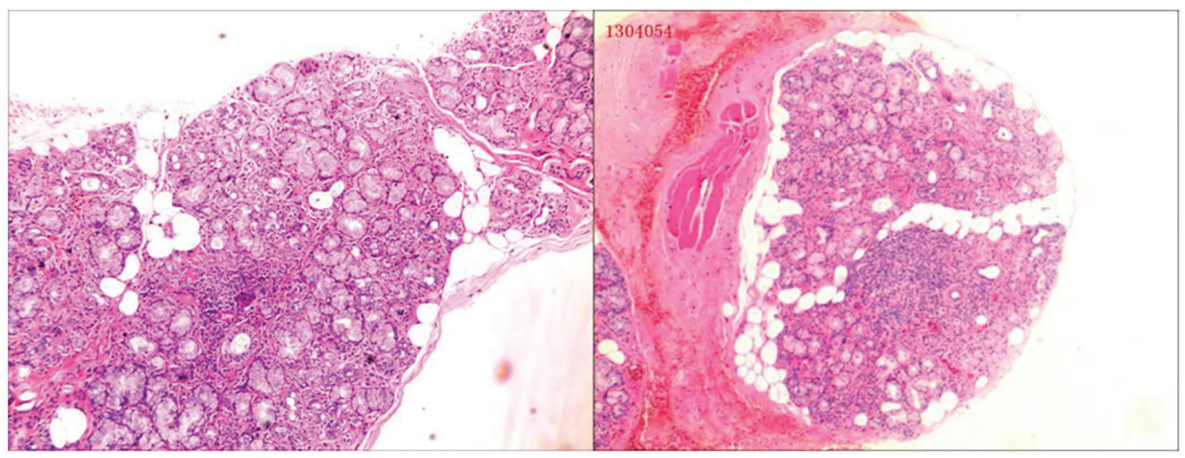

Figure 5. Labial salivary gland biopsy showed two foci of focal lymphocytic sialadenitis. 
disseminated encephalopathy also needs vigilance. Earlier recognition and treatment for this situation is important.

\section{References}

1. Binard A, Devauchelle-Pensec V, Fautrel B, Jousse S, Youinou P, Saraux A. Epidemiology of Sjogren's syndrome: where are we now? Clin Exp Rheumatol. 2007;25(1):1-4.

2. Fox RI. Sjogren's syndrome. Lancet. 2005;366(9482):321331.

3. Shiboski SC, Shiboski CH, Criswell L, Baer A, Challacombe S, Lanfranchi H, Schiodt M, et al. American College of Rheumatology classification criteria for Sjogren's syndrome: a data-driven, expert consensus approach in the Sjogren's International Collaborative Clinical Alliance cohort. Arthritis Care Res (Hoboken). 2012;64(4):475487.
4. Bournia VK, Diamanti KD, Vlachoyiannopoulos PG, Moutsopoulos HM. Anticentromere antibody positive Sjogren's Syndrome: a retrospective descriptive analysis. Arthritis Res Ther. 2010;12(2):R47.

5. Gono T, Kawaguchi Y, Katsumata Y, Takagi K, Tochimoto A, Baba S, Okamoto Y, et al. Clinical manifestations of neurological involvement in primary Sjogren's syndrome. Clin Rheumatol. 2011;30(4):485-490.

6. Massara A, Bonazza S, Castellino G, Caniatti L, Trotta F, Borrelli M, Feggi L, et al. Central nervous system involvement in Sjogren's syndrome: unusual, but not unremarkable - clinical, serological characteristics and outcomes in a large cohort of Italian patients. Rheumatology (Oxford). 2010;49(8):1540-1549.

7. Salliot C, Gottenberg JE, Bengoufa D, Desmoulins F, Miceli-Richard C, Mariette X. Anticentromere antibodies identify patients with Sjogren's syndrome and autoimmune overlap syndrome. J Rheumatol. 2007;34(11):22532258. 